\title{
Super Enhancer Inhibitor GZ17-6.02
}

National Cancer Institute

\section{Source}

National Cancer Institute. Super Enhancer Inhibitor GZ17-6.02. NCI Thesaurus. Code C158082.

A synthetic formulation of the Arum palaestinum plant that has been fortified with the already naturally occurring constituents of isovanillin, linolenic acid, and beta-sitosterol, with potential antineoplastic activity. Upon oral administration, GZ17-6.02 may induce apoptosis through caspase-3 activation and poly(ADP-ribose) polymerase (PARP) cleavage, inhibit tumor cell progression by attenuating macrophage infiltration, and inhibit the phosphorylation of several mediators of tumor cell proliferation including Src kinase, extracellular signal-regulated kinases 1 (ERK1) and 2 (ERK2), epidermal growth factor receptor (EGFR), serine/threonine protein kinase AKT (protein kinase B), signal transducer and activator of transcription 2 (STAT-2), and serine/threonine-protein kinase Chk2 (Chk2). GZ17-6.02 may also inhibit certain super enhancers (SEs) that play an important role in the regulation of the sonic hedgehog (SHH) pathway and cancer stem cell activity. Super enhancers (SES) are unique areas of the genome that are densely bound by numerous transcription factors and play a pivotal role in the cell, including tissue specification, identity and maintenance. SEs are known to regulate the expression of associated genes and often drive high-level transcription. 